\title{
Analysis of Tencent's Successful Development Strategy
}

\author{
Junxiong Huang
}

\author{
Dongguan Camford Royal Academy, Dongguan, CHINA 529500 \\ 2920399135@qq.com
}

\begin{abstract}
Tencent has changed from a small company to an international company with billions of dollars in assets. This article further discusses Tencent's overall development and Tencent's business decisions by analyzing Tencent's marketing strategy. This paper will further analyze Tencent's strategies by referring some materials of Tencent's background and development history. Finally, the author found that Tencent's strategy is good at using innovative technology to dig out some other large companies that have no functions but will win theconsumers. After Tencent gains a consumer base, the company's leaders know how to analyze some market conditions to improve new products and functions, and gradually expand the scope of sales. These decisions are indeed innovative and wise.
\end{abstract}

Keywords: Tencent, innovation, development strategy, QQ, giant enterprises

\section{INTRODUCTION}

The development of new industries is inseparable from innovation and product marketing. Only good products and excellent strategies can attract consumers. In today's world, many emerging industries have gone bankrupt or experienced fiscal deficits before they began to develop.

Tencent just imitated foreign ICQ companies and developed in China. However, Tencent has made good use of some development strategies and marketing methods in China. In the process of Tencent's development, some large companies have lost the market, such as MSN, which occupied the most market share in the beginning. But MSN did not continue to attract users, it failed in the competition This paper mainly aims to learn more about market policies that can help small companies develop better by studying the market strategy of Tencent, which was once considered as a world-famous small company. This study will focus on the history of Tencent and their process of the development of some typical or compelling business decisions for the sake of make a reference to the leaders of companies.[1] The author collected some statistical charts and data on the Internet to better understand the competitive environment and methods of Tencent and how the difficulties encountered in their development had been solved. There are many strategies that Tencent leverage to success.So the author will outline the development of this copycat company, from its beginning to its success. During this period, many companies have failed in the competition with Tencent, and these strategies used by Tencent can be used for reference by other companies in their future business decisions. The author can also further help people understand the importance of marketing strategy for a company and what kind of marketing strategy is successful. These smart skills should be used in business negotiation and business analysis in the future, so that decision makers can operate a company in a success way .

\section{TENCENT'S STRATEGY TO SURVIVE FROM GIANT ENTERPRISES}

\subsection{Strategy1 :appropriate market}

As early as 1998, Tencent had developed QICQ (later renamed QQ for copyright reasons) after its founding. At that time, there was a very good background that the number of Internet users in China had increased to a certain extent in 2000, which meant the importance of their QICQ software release. [2] Because there will be more consumers who need to communicate online, and QICQ can meet their needs. But at that time, China lacked Internet software like ICQ. Also, it can be seen from figure 1, the number of Internet users in China is increasing every year, which clearly shows that Chinese people are using the Internet more and more, so the launch of software is a necessary business opportunity.so Tencent seize the business opportunity and created a QICQ in China using the model of foreign ICQ. 


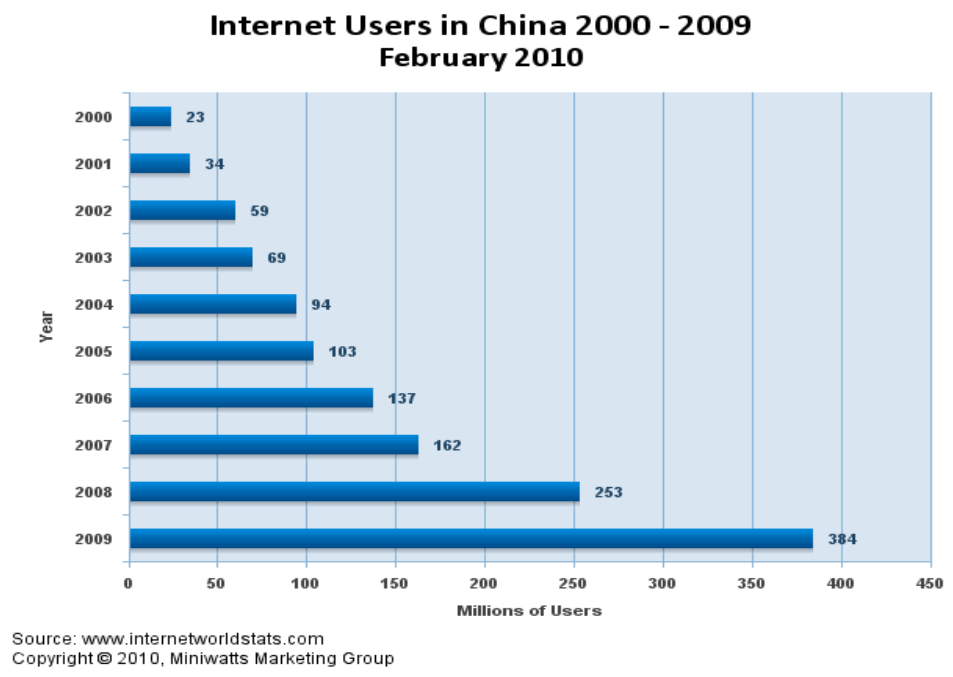

Figure 1 CHINA internet users population statistics

\subsection{Strategy2:innovation}

The reason why QQ can compete with ICQ is closely related to its innovation. ICQ is an Internet concept, while QQ is a software concept. The reason for ICQ failure is that all ICQ information is stored on the user side. The second is that ICQ makes money from instant messaging software tailored to businesses. In this case, the concept of affirmation QQ will be more attractive to customers. The second innovative concept is group chat, in the rise of QQ in that era, "chat rooms" can be said to be necessary online. Among them at that time the "chat room" of the use of the maximum peak time, a room of the chat room has tens of thousands of people online at the same time.QQ seized the opportunity. [3]In August 2002, Tencent released a new version of QQ, adding a new feature to become the enemy of chat rooms. Among them, the QQ group function quickly turned QQ into a real social network platform, and the once-popular chat room quickly collapsed into recession. The shift allows online users to actually get together and communicate with people they want to talk to and be familiar with rather than with strangers and people they do not know.

\subsection{Strategy3: Operate branches}

The first version of OICQ was released in 1999, and by 2004 it had captured $78 \%$ of the instant messaging market in China.[4] At that time, there appeared a big MSN company to block the emergence of QQ, MSN is Microsoft's social software, and once was the world's largest instant messaging software. When MSN first entered the Chinese market, without any publicity or localization support, MSN easily took the second place with $10 \%$ of the Chinese instant messaging market. And the MSN user group is mainly concentrated in the lofty business people, the number of people already more than 10 million. MSN was targeted at business people with a relatively single user group, while QQ was targeted at young people of different classes. In fact, the soft formation of MSN in China r\&d center, ready to implement localization, at that time, when people thought Tencent will quit the market MSN first reach channel to the society in the form of contract management bidding, then quickly cut into the telecom value-added services, a deal with yahoo, almost will be around half the world's global communications users in their own hands. Despite this QQ also did not own and MSN alliance, but began to test their product to the heart of high-end users, Tencent launched enterprise edition QQTM, and through the file Fixed-point continuingly, message communication, video conference and a series of technical innovations, a series of technology, and the function of the MSN did not chat with a stranger, which this small feature seemingly insignificant, but it caused a chance to QQ to strike the MSN to lose their market share .[5] MSN has created such a large market share, is not willing to make a change, and their internal management issues make QQ opportunity more and more, in the end by 2008, Microsoft's market share amount is only four percent, compared with the QQ who own more than one hundred million subscribers led to Microsoft announced to abandon the Chinese MSN market.

\subsection{Develop more service ranges}

After establishing the market, Tencent gradually expanded into more businesses, and in the early 2010s, many Chinese domestic companies began producing inferior smartphones with many additional features. The main advantage of domestic smartphones over popular global brands is their affordable price. 


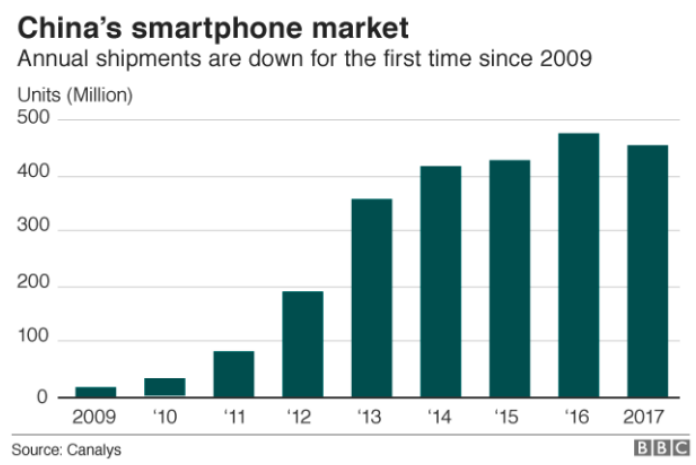

Figure 2 China's smartphone market amount

According to figure 2, it is obvious from the chart that the mobile phone market in China each year had an extremely big enhancement which reached peak in 2016, despite a small amount decrease in 2017.[6]But overall, the surge of the whole mobile phone market is in this also means that consumers can more easily buy a reasonable price and accept the mobile phone. With the support of the mobile phone market, Tencent gradually launched Wechat, the software, and added more novel functions such as: WeChat Pay, Mini Programs, and Game Center are very popular among consumers. In 2010, digital content subscriptions, membership subscriptions, and sales of virtual goods became Tencent's main sources of revenue, accounting for more than $70 \%$ of total revenue. In 2013, Tencent slowly turned to mobile games. Mobile QQ and WeChat launched a game center, which has a huge mobile game user base. [7] In the following years, the company focused on the global game market, buying stakes or aggressively buying overseas game companies to become the world's largest online game company, controlling 13 percent of the market. [8]Its gaming revenue broke the $\$ 10$ billion mark in fiscal 2016. It can be clearly seen from figure 3 that Tencent's game revenue has been increasing since 2010, which clearly shows that Tencent has achieved very huge revenue in the game industry.

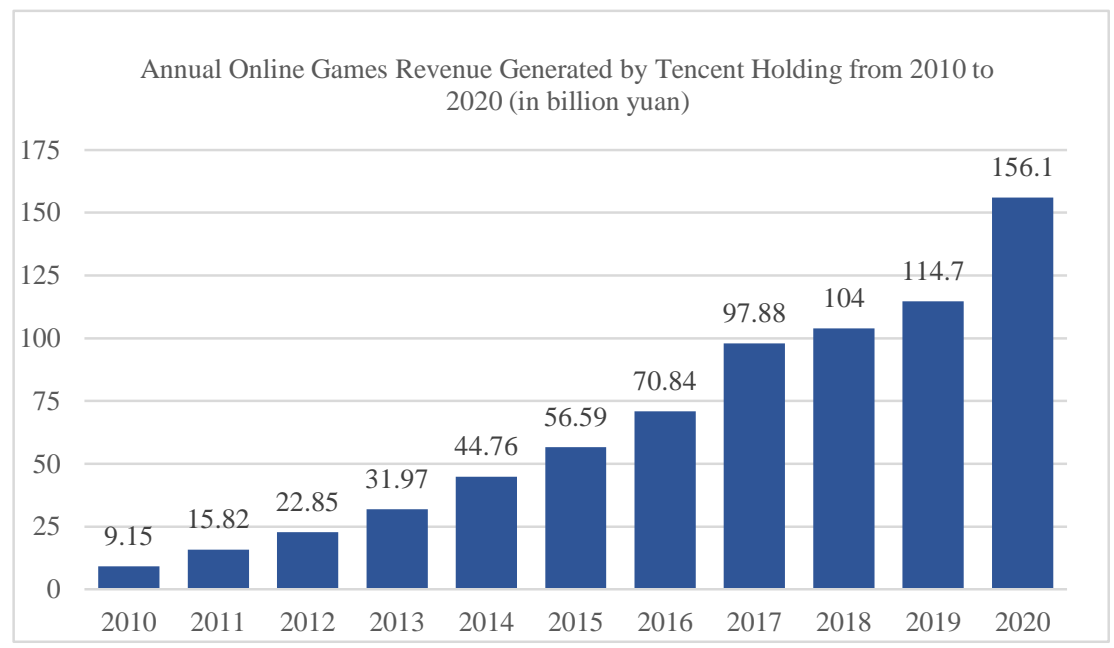

Figure 3 The statistics of games revenue created by Tencent from 2010-2020

\section{DISCUSSION}

In fact, Tencent also has some limitations. After too many branches are created, some subsidiaries are under great pressure in competition with similar companies. [9]For example, Weishi will face immature macro environment and its content is not attractive enough to the platform.[10]Tencent video, a sub corporation of Tencent also meet some challenges of the limitation of source videos.[11]Tencent's own applicatio also have limitations, such as wechat's payment function, in which users are not accounts, resulting in small transaction quotas, so consumers can only regard wechat as a tool for small purchases or transfers. So, not only from Tencent's sub company or their invented applications are all facing a lot of challenges and restrictions. The author believes that the Internet has a lot of innovation opportunities after all future cannot be confined to doing software or pure service and Tencent have to do is "connector", the service and user to connect to stand up, for example using the 
Internet to connect the energy, they need to put some unrelated fields by connecting to better benefit some consumers or country. For example: medical care, transportation. In addition, the author believes that Tencent needs to keep pace with The Times. As a company with a large consumer base, it needs to cater to consumers' needs and invent more things that consumers like, such as new energy vehicles, which are very popular recently. Moreover, security index, for some Internet fraud and some infrastructure, as well as payment and other areas, Tencent needs to maintain long-term investment to prevent their customers from being cheated.

\section{CONCLUSION}

After analyzing Tencent's development strategy, the author found that Tencent's success comes from its innovation and wise development decisions. Therefore, when Tencent emerged as an industry based on imitation, it had already faced many difficulties. As for MSN, a giant company that had already occupied the market, Tencent used some innovative means to defeat them. After occupying the market, Tencent still knew how to attract customers and find the right market opportunities. This just finally makes Tencent this company finally can establish a foothold in the market.In the process of research, this article only analyzes part of Tencent's business and strategies, and the selected data also has certain limitations. In subsequent research, the author will further explore the development of Tencent through quantitative analysis and other methods.

\section{REFERENCES}

[1]Bhatia, A. (2020). Interesting facts you never knew about Pony Ma, the force behind Tencent. The Indian Express. https://indianexpress.com/article/technology/technews-technology/interesting-facts-about-pony-mathe-ceo-of-chinese-internet-behemoth-tencent6585070/. [2]Cantale I. (2020, August 27). How tencent became the world's most valuable social network firm - with barely any advertising. https://theconversation.com/how-tencent-becamethe-worlds-most-valuable-social-network-firmwith-barely-any-advertising-90334.

[3]Heinrich, R. \&. (2020, October 17). Tencent's innovation strategy. Medium. https://renheinrich.medium.com/tencents-innovationstrategy-37547aec491c.

[4]SOU, H. U. (2018, November 22). Tencent QQ had 3 competitors, 2 of which were from abroad, and they were all easily defeated. https://www.sohu.com/a/277172284_450537.

[5]AI, Y. O. N. G. L. I. A. N. G. C. H. A. O. J. I. C. H. A. N. P. I. N. (2019). Ai Yongliang: Why did MSN lose in the competition between QQ and MSN? . https://baijiahao.baidu.com/s?id=16376246916874 $51161 \& w f r=$ spider $\&$ for $=$ pc.

[6]Guo, B., \&amp; Liu, J. (2021, September 6). Internet companies' cultural entrepreneurialism and Policy interactions in China: TENCENT'S case Of "NEOCULTURE creativity" strategy. Digital Business. https://www.sciencedirect.com/science/article/pii/S 2666954421000144.

[7]Li, P. (2021, March 24). Tencent's quarterly revenue jumps as online Gaming surges. Reuters. https://www.reuters.com/article/us-tencent-resultsidUSKBN2BG10B.

[8]Thomala, L. L. (2021, April 19). Tencent: Online games REVENUE 2020. Statista. https://www.statista.com/statistics/527280/tencentannual-online-games-revenue/.

[9]JIN, X. (2021).The Dilemma and Outlet of Tencent Microvision,Journal of News Research Guide, https://www.cnki.com.cn/Article/CJFDTotalXWDK202101102.html

[10]GU, YX (2019). Tencent Video's Countermeasures to Cope with the Dilemma,Northern Media Research, https://www.cnki.com.cn/Article/CJFDTotalBMYJ201906015.htm.

[11]ZHANG,MX (2014).Tencent's Risks and Alibaba's Challenges. 04 , 2014. http://www.cqvip.com/QK/82514X/201404/49528 653.html 